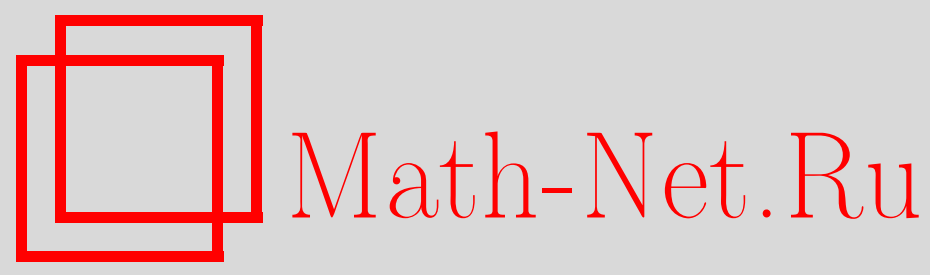

В. О. Мантуров, Скобочная полугруппа узлов, Матем. заметки, 2000, том 67, выпуск 4, 549-562

DOI: https://doi.org/10.4213/mzm870

Использование Общероссийского математического портала Math-Net.Ru подразумевает, что вы прочитали и согласны с пользовательским соглашением http://www . mathnet.ru/rus/agreement

Параметры загрузки:

IP : 54.164 .48 .24

26 апреля 2023 г., 10:52:32

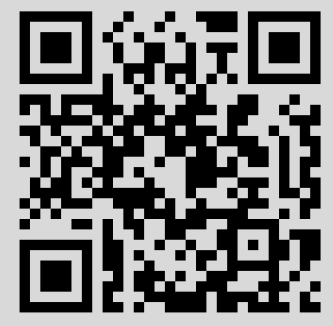




\title{
СКОБОЧНАЯ ПОЛУГРУППА УЗЛОВ
}

\author{
В. О. Мантуров
}

Статья посвящена кодированию зацеплений с отмеченной точкой на ориентированной компоненте с помощью правильных двухскобочных структур - некоторых слов в алфавите из (, ), [ и ]. Таким образом, естественно задается полугруппа узлов, полугрупповой операцией в которой является конкатенация, а классы эквивалентности получаются факторизацией по так называемым "глобальным соотношениям". Важным шагом построения данной полугруппы является кодирование зацеплений с помощью так называемых $d$-диаграмм.

Библиография: 6 названий.

\section{§1. Введение}

В настоящей работе предлагается новьй способ кодирования зацеплений в виде слов в алфавите из [, ], (, ). В настоящей статье под словом зацепление понимается зацепление с выделенной ориентированной компонентой и выбранной точкой на ней, в частности, ориентированньй узел с отмеченной точкой.

Основной идеей построения такого кодирования является геометрическая конструкция соответствия между зацеплениями и так назьваемьми атомами и $d$-диаграммами (см. [1], [2]), которая позволяет дать простую комбинаторную кодировку зацеплений. Это кодирование порождает простое алгебраическое описание полугрупшы узлов, т.е. зацеплений в указанном вьше смысле: элементы полугрушы представляются в виде классов эквивалентности некоторых слов в указанном алфавите. Опишем кодирование и эту полугрупш.

Геометрически полугруппа узлов $K$ описывается следующим образом.

i) Элементами $K$ являются изотопические классы зацеплений с отмеченной компонентой, ориентацией данной компоненты и выделенной точкой на ней.

ii) Единицей данной полугруппы является класс тривиального узла.

iii) Произведение двух элементов $A$ и $B$ определяется следуюшим образом. Пусть $A^{\prime}$ и $B^{\prime}$ - представители классов $A$ и $B$. Пусть $A_{1}, A_{2}$ - точки на $A^{\prime}$ в малой окрестности начальной (отмеченной) точки, причем $A_{1}$ находится после $A_{2}$ отностительно ориентации компоненты. Аналогично выберем $B_{1}, B_{2}$ на зацеплении $B^{\prime}$. Расположим $A^{\prime}$ и $B^{\prime}$ в пространстве незацепленным образом так, чтобы начальные точки зацеплений $A^{\prime}$ и $B^{\prime}$ находились в на близком расстоянии. Кроме того, потребуем, чтобы пересечение зацеплений $A^{\prime}$ и $B^{\prime}$ с малой окрестностью $U$ соответствующих отмеченных точек представляло собой два параллельных отрезка. Потребуем также, чтобы существовала прямая $l$ на плоскости проекции, не пересекающая проекции $A^{\prime}$ и $B^{\prime}$, т.е. проекции 
$A^{\prime}$ и $B^{\prime}$ лежали по разные стороны от $l$. Удалим из $A^{\prime}$ дугу $A_{1} A_{2}$, а из $B^{\prime}$ дугу $B_{1} B_{2}$ и соединим отрезками точки $A_{1}$ с $B_{2}$, а $B_{1}$ с $A_{2}$. Класс получившегося зацепления, в котором за начальную точку выбрана $A_{1}$, а ориентация в окрестности этой точки совпадает с ориентацией $A$ и $B$, назовем произведением $A B$ (см. рис. 1$)$.
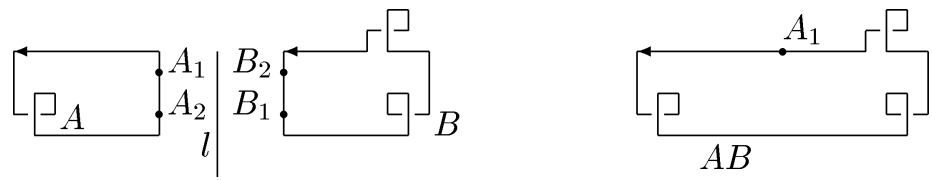

Рис. 1. Произведение зацеплений

ЗАмЕчАниЕ. Вообще говоря, произведение зацеплений не зависит от выбора отмеченной точки, а лишь от выбора ориентированной компоненты.

ОПРЕДЕЛЕНИЕ 1.1. Назовем слово $A$ в алфавите из четырех скобок (, ), [ и ] npaвильной двухскобочной структурой, если для каждого начального подслова количество открьвающихся квадратных скобок неменьше количества закрывающихся квадратных скобок, а количество открьваюшихся круглых скобок не меньше количества закрываюшихмя круглых скобок и при этом для самого слова $A$ эти неравенства превращаются в равенства.

Определим полугрупшу $G$ правильных двухскобочных структур следующим образом.

i) Элементами полугруппы $G$ являются классы эквивалентности правильных двухскобочных структур по соотношениям (1)-(7), перечисленным ниже. Эти соотношения мы будем называть глобальными .

Каждое из соотношений (2)-(6) означает, что если некоторое слово $I$ является правильной двухскобочной структурой, а некоторое подслово слова $I$ имеет вид, описанный в правой (левой) части глобального соотношения, то правильная двухскобочная структура $J$, полученная из $I$ заменой этого подслова на подслово, стоящее в левой (соответственно правой) части глобального соотношения, эквивалентна $I$. Соотношения считаются применимьпи лишш в случае правильности двухскобочных структур в обеих частях равенств, при этом правильность скобочной структуры в одной части равенства влечет правильность скобочной структуры в другой части равенства.

(1) ()$=[]=1$.

(2) $\left(\left[\right.\right.$ ) $\left.A_{1} A_{2}\right]=\left([)\left(A_{2} A_{1}\right)^{\prime}\right]$, где одно из слов $A_{i}, i=1,2$, имеет правильную скобочную структуру относительно $()$, при этом штрих означает, что если некоторая скобка [ из $A_{1}$ закрьвалась скобкой ] из $A_{2}$, то в $A_{2} A_{1}{ }^{\prime}$ соответствующая скобка в $A_{2}$ открьвающаяся, а скобка в $A_{2}$ закрывающаяся.

(3) ( [ ) ( ] $A)=A$, где слово $A$ имеет правильную относительно (, ) структуру.

(4) $([(A)) B]=[([A) B]]$, где слова $A$ и $B$ имеют правильные $($,$) -структуры.$

(5) $A[((] B) C)=A((B[[)]](C)[[$ ] ] , где слова $A, B, C$ имеют правильные [, ]-структуры.

(6) $A B C D=\left(\left[\right.\right.$ ) $\left.A\left[(] D^{\prime}\right] C^{\prime}\right) B^{\prime}$, где слово $A D$ имеет правильную [,]-структуру, а слово $D C$ - правильную (, )-структуру. При этом $B^{\prime}, C^{\prime}, D^{\prime}$ означают, что скобки, открьвающиеся в одном слове и закрьвающиеся в другом, меняются местами. Например, если скобка [ из $B$ закрывалась в слове в левой части скобкой ] из $C$, то в слове в правой части открывающаяся скобка будет в $C$, а закрьвающаяся - в $B$. 
(7) $A=A^{*}$, где $A-$ слово с правильной двухскобочной структурой, а $A^{*}-$ слово с правильной двухскобочной структурой, получаемое из $A$ заменой круглых скобок на квадратные, и наоборот.

ii) Единицей полугрупшы $G$ является пустое слово.

iii) Полугрупповая операция в $G$ задается приписыванием (конкатенацией).

Главньй результат работы состоит в построении канонического изоморфизма алгебраической полугруппы $G$ и геометрически определенной полугрупшы узлов $K$ (доказательство изомофности см. в разделе 2.4).

Важным шагом построения такого изоморфизма является трактование полугруппы $K$ в терминах так назьваемых $d$-диаграмм.

Интересной задачей является нахождение полугрупшы $H$, изоморфной $G$, элементами которой являлись бы классы эквивалентности некоторых слов в конечном алфавите по локальным соотношениям. Локальным соотношением мы назьваем тождество пары слов $a$ и $b$, т.е. соотношение в обычном смысле в полугруше, заданной образуюшими и соотношениями. При этом факторизацией полугруппы по такому соотношению назьвается полугруппа, полученная из исходной отождествлением слов $P_{a}$ и $P_{b}$, одно из которых получено заменой конечного числа независимых вхождений подслова $a$ на $b$.

В последнем разделе статьи мы рассматриваем виртуальные узлы и смотрим, в какой мере наши геометрические конструкции здесь применимы. Основными понятиями, использованньци в этой работе, являются движения Райдемайстера (см., например, [3]), высотные атомы (в смысле [2]) и хордовые диаграммы (рассматриваемые как комбинаторный объект, безотносительно к сингулярным узлам [2]).

\section{§2. Построение полугруппы $G$}

2.1. Основные понятия. Следуя [2], [4], [5], дадим определения атома, высотного атома и $f$-графа, которые будут необходимы для построения полугрупшы $G$.

ОПРЕДЕЛЕНИЕ 2.1.1. Атомом (понятие введено А.Т. Фоменко [4]) назьвается пара $\left(f^{-1}(c-\varepsilon, c+\varepsilon), f^{-1}(c)\right)$, где $f$ - некоторая функция Морса на замкнутом двумерном многообразии $M, c$-ее критический уровень, $\varepsilon$ - некоторое малое число такое, что внутри $f^{-1}(c-\varepsilon, c+\varepsilon)$ нет критических точек функции $f$, кроме точек, лежащих на критическом уровне.

ЗАмЕчАниЕ. Фактически для определения атома нужно знать поведение функции $f$ не на всем $M$, а лишш на первой компоненте атома.

Вторая компонента атома (критический уровень) называется остовом атома. Остов представляет собой граф кратности 4.

В дальнейшем послекритическим уровнем атома будем называть набор окружностей $f^{-1}(c+\varepsilon)$.

Атом называется ориентируемым, если его первая компонента ориентируема как многообразие с краем.

Обычно атомы рассматриваются с точностью до естественной эквивалентности, которая называется изоморфизмом, а именно, два атома назьваются изоморфнымии, если существует диффеоморфизм первых компонент атомов, отображающий вторую компоненту одного атома на вторую компоненту другого атома и сохраняющий направление роста функций. 
ОПРЕДЕЛЕНИЕ 2.1.2. Высотны.м атомом [2] назьвается атом, изоморфньй атому, задаваемому функцией высоты на некотором многообразии, вложенном в $\mathbb{R}^{3}$.

ВАЖНОЕ ЗАмЕЧАНИЕ. Высотный атом всегда является ориентируемьм.

Рассматривая атом с точностью до изоморфизма, его можно задавать с помощью остова со специальной структурой, определяемой следующим образом.

ОПРЕДЕЛЕНИЕ 2.1.3. Говорят, что граф (остов атома) снабжен $B$-структурой, если четыре полуребра, выходящих из каждой вершины, разбиты на две пары ребер, называемых противоположными, а также в каждой вершине выбраны две пары соседних (не противоположных) ребер.

Остов с $B$-структурой однозначно задает некоторый атом, при этом $B$-структура также назьвается $B$-структурой атома.

В дальнейшем будем назьвать такой выбор $B$-структурой атома.

ЗАмЕчАниЕ. На рис. 2 пары точек в окрестности вершин задают $B$-структуру атома, точнее, обозначают пары соседних ребер или углов послекритического уровня.

ОПРЕДЕЛЕНИЕ 2.1.4. Назовем $d$ - диаграммой хордовую диаграмму (см., например, [6]), хорды которой можно разбить на два семейства непересекающихся хорд.

\section{2. Построение соответствия меж,ду зацеплениями и $d$-диаграммами.}

Для построения соответствия между $d$-диаграммами и зацеплениями нам потребуются определения и результаты, полученные в [5], [2], [1].

Легко показать [2], что атом явялется высотным тогда и только тогда, когда его остов вложим в плоскость таким образом, что заданный и индуцированньй порядки противоположности полуребер в каждой вершине совпадают.

Соответствие между высотными атомами и зацеплениями определяется следующим образом [2]. Пусть дан высотньй атом $V$. Вложим его остов в плоскость таким образом, чтобы сохранялось отношение противоположности полуребер в каждой вершине (структура $A$ ). В каждой вершине вложенного остова сходятся четыре полуребра. Мы превратим этот остов в диаграмму зацепления, соответствующую атому, указав, какая пара противоположных ребер задает проход (и какая переход). Это определяется правилом: при повороте от ребра “прохода" к ребру “перехода", соседнего с ним, движение происходит по часовой стрелке.

Теорема об атомах и узлах [2] утверждает, что при различных вложениях в плоскость остова одного и того же высотного атома с сохранением противоположности полуребер в каждой вершине получаются диаграммы изотопных зацеплений.

Таким образом, изотопический класс зацепления определяется по высотному атому однозначно.

ОПРЕДЕЛЕНИЕ 2.2.1. Назовем такое зацепление защеплением, соответствующим высотному атому $V$. Все высотные атомы, которым соответствует некоторое зацепление $K$, назовем атомами, порождаемыми зачеплением $K$.

Определена и обратная операция: по каждой плоской диаграмме зацепления строится некоторьй высотный атом.

Каждому ориентируемому атому взаимно однозначно соответствует так назьваемая полихордовая диаграмма - диаграмма, состоящая из нескольких ориентированных окружностей и хорд, соединяющих точки на окружностях (всего конечное число хорд, при этом каждая точка на окружности инцидентна не более чем одной хорде; см. 
рис. 2). Пусть дан ориентируемый атом $V$. Полихордовая диаграмма $D$, соответствующая атому $V$, строится следующим образом. Рассмотрим “послекритические окружности" атома $V$, т.е. замкнутые кривые, лежащие чуть выше критического уровня соответствующей функции, и ориентируем их согласованно. В окрестности каждой вершины атома $V$ поставим по хорде, соединяющей дуги послекритических окружностей. Полученньй граф, состоящий из окружностей и хорд, назовем полихордовой диаграммой, соответствующей атому $V$.

ЗАмЕЧАнИЕ. Зацепление, соответствующее атому на рис. 2, является тривиальным зацеплением двух окружностей с двумя перекрестками.

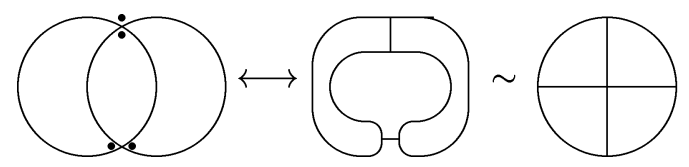

Рис. 2. Атом и соответствующая (поли)хордовая диаграмма

Неориентируемым атомам аналогичньм образом соответствуют диаграммы с метками на ребрах (см. также [5], понятие $f$-графа).

ТЕОрема 2.2.1. Для каждого изотопического класса неориентированного классического зачепления $K$ без отмеченных точек существует порождаемый им высотный атом $V$ такой, что полихордовая диаграмма, соответствующая $V$, является диаграммой с одной окружностью, т.е. обычной хордовой диаграммой.

ДокАЗАТЕЛЬСТВО заключается в многократном изменении данной диаграммы с несколькими окружностями с помощью вторых движений Райдемайстера, при каждом из которых количество окружностей уменьшается на единицу, а в конце становится равным единище.

Теорема 2.2 .2 [1]. Если атому $V$ соответствует классическая хордовая диаграмма $C$, то (атом $V$ высотный) $\Longleftrightarrow($ аиграмма $C$ является $d$-диаграммой)

Итак, теперь можно переходить к построению полугруппы $G$. Мы сначала построим изоморфную ей полугруппу $D$ в терминах $d$-диаграмм, а затем полугруппу $G$ с помощью правильных двухскобочных структур.

Пусть дано зацепление $K$ с выделенной точкой. Поставим ему в соответствие множество $d$-диаграмм с отмеченной точкой таких, что высотные атомы, соответствующие этим диаграммам, порождают зацепление $K$, а выделенная точка зацепления $K$ естественным образом соответствует выделенной точке на $d$-диаграмме. В соответствие $d$-диаграмме с пустьм множеством хорд мы поставим тривиальньй узел.

Из сказанного вьше следует, что каждому зацеплению с отмеченной точкой соответствует хотя бы одна $d$-диаграмма, при этом каждой $d$-диаграмме с отмеченной точкой можно поставить в соответствие зацепление с отмеченной точкой.

ОПРЕДЕЛЕНИЕ 2.2.2. Назовем две $d$-диаграммы әквивалентнымии, если они отвечают изотопным зацеплениям. 
ОПРЕДЕЛЕНИЕ 2.2.3. Пусть $D$ - полугруппа всех классов эквивалентности $d$-диаграмм с отмеченной точкой; полугрупповую операцию в $D$ определим следующим образом: пусть даны две $d$-диаграммы $D_{1}$ и $D_{2}$, заданные как наборы пар точек на окружности, соединяемых хордами и задаваемых углами между радиус-векторами и осью $O x$, через $x_{1, i}, x_{2, i}$ обозначим координаты хорд $D_{1}$, а через $y_{1, i}, y_{2, i}-$ координаты хорд $D_{2}$. Тогда диаграмму, хорды которой соединяют точки с углами $x_{1, i} / 2, x_{i, 2} / 2$, а также $y_{1, i} / 2+\pi, y_{i, 2} / 2+\pi$ назовем произведением $D_{1} D_{2}$ (рис. 3 ).

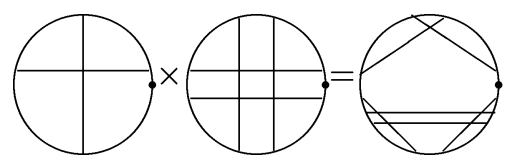

Рис. 3. Произведение $d$-диаграмм

По построению получаем следующую теорему.

TеОрема 2.2.3. Полугруппа $K$ изоморфна полугруппе $D$.

2.3. Трактовка $d$-диаграмм в терминах правильных двухскобочных структур. Рассмотрим некоторую $d$-диаграмму $D$ и произвольное разбиение множества ее хордна два подмножества непересекающихся хорд. Начнем движение от начальной точки диаграммы $D$ вдоль по окружности против часовой стрелки. Из двух вершин каждой хорды назовем первой ту, которая встречается раньше при движении против часовой стрелки.

Каждьй раз встречая первую вершину хорды, будем писать открьвающуюся скобку, а встречая конец хорды - закрывающуюся. При этом для хорд первого семейства будем использовать круглые скобки, а для хорд второго семейства - квадратные. Возвратившись в начальную точку, получим слово $A(D)$. Легко видеть, что $A(D)$ является правильной двухскобочной структурой

ОПРЕДЕЛЕНИЕ 2.3.1. Назовем все такие структуры $A(D)$ правильными скобочными структурами, соответствующими хордовой диаграмме $D$ с отмеченной точкой.

ПРИМЕР. См. рис. 4.

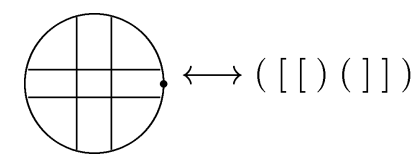

Рис. 4. Соответствие $d$-диаграмма $\longleftrightarrow$ правильная двухскобочная структура

Легко видеть, что и обратно каждая правильная двухскобочная структура порождает некую $d$-диаграмму с отмеченой точкой.

Рассмотрим зацепление $K$ с отмеченной точкой.

ОПРЕДЕЛЕНИЕ 2.3.2. Пусть $K$ - зацепление, a $\{D\}$ - множество всех $d$-диаграмм с отмеченной точкой, соответствующих этому зацеплению. Каждую из скобочных структур, соответствующих $d$-диаграммам из $\{D\}$, будем также назьвать правильной двухскобочной структурой, соответствующей зачеплению $K$. 
Таким образом, имеют место следующие факты:

1) все зацепления кодируются правильными двухскобочными структурами;

2) каждой правильной двухскобочной структуре соответствует зацепление;

3) пустому слову соответствует класс тривиального зацепления;

4) полугрупповой операции в полугруппе $K$ соответствует приписьвание одной правильной двухскобочной структуры к другой.

ОПРЕДЕЛЕНИЕ 2.3.3. Назовем две правильные двухскобочные структуры, порождающие изотопные зацепления, әквивалентными.

ПРИМЕРЫ. В полугрупе $G$ правьй трилистник задается в данной полугруппе как ( [ [ [ [ ) ] ] ] ] (см. рис. 5), левьй - как ( ( [ [ ) ) ] ].
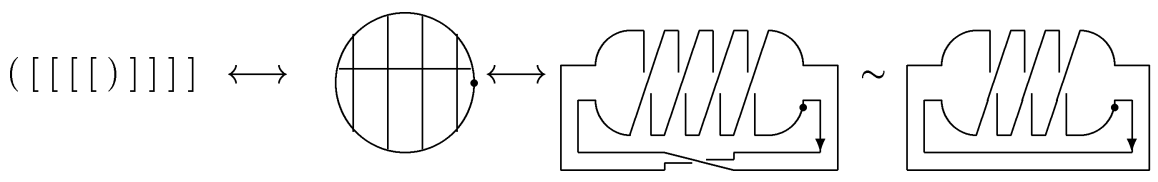

Р ис. 5. Правый трилистник

ЗАмЕчАнИЕ. Каждое классическое зацепление может быть задано с помощью прямоугольной ломаной петли внутри первого квадранта плоскости $O x y$. Петля выходит из $(0,0)$ и входит в $(0,0)$, и все ее звенья являются единичными отрезками, горизонтальными или вертикальными. При этом каждая такая петля задает некоторое зацепление.

Действительно, каждую правильную двухскобочную структуру можно трактовать как такую петлю, где ( означает один шаг вправо, ) - влево, [- вверх, а ] - вниз.

2.4. Доказательство изоморфности $G$ и $K$. Итак, мы знаем, что существует биекция между изотопическими классами зацеплений и классами эквивалентности правильных двухскобочных структур, при этом полугрупповая операция в полугруппе $G$ соответствует полугрупповой операции в полугрупе $K$. Остается лишь доказать, что две правильные двухскобочные структуры эквивалентны тогда и только тогда, когда одна из них переводится в другую с помощью конечной последовательности глобальных соотношений (1)-(7), описанных во введении.

Очевидно, что две правильные двухскобочные структуры порождают одну и ту же $d$-диаграмму тогда и только тогда, когда они переводятся друг в друга движением (7).

Поэтому для доказательства главной теоремы осталось проверить следующий факт.

УТВЕРЖДЕНИЕ 2.4.1. Две d-диаграммы $A$ и $B$ задают класс одного и того же зачепления тогда и только тогда, когда существуют соответствующие правильные двухскобочные структуры а и б и последовательность глобальных преобразований (1)-(6), переводящих а в $b$.

Для доказательства этого утверждения нужно рассмотреть движения Райдемайстера и выделить из них такие композиции, которые оставляют диаграмму зацепления в классе диаграмм, соответствующих монохордовых диаграмм, т.е. полихордовым диаграммам с одной окружностью. Впоследствии окажется, что они как раз представляют собой движения (1)-(6).

По сути дела, нам надо выбрать в множестве комбинаций всех движений Райдемайстера множество всех движений, оставляющих диаграмму монохордовой (классической). 
2.4.1. Первое движение Райдемайстера. Рассмотрим первое движение Райдемайстера диаграмм зацеплений как изменение соответствующих (поли)хордовых диаграмм.

Если в начальном положении было $n$ окружностей, то после этого движения либо к одной окружности добавляется отдельно стоящая хорда, либо добавляется еще одна окружность, из которой выходит всего одна хорда, соединяющая ее с окружностью из числа $n$ уже имеюшихся (см. рис. 6).

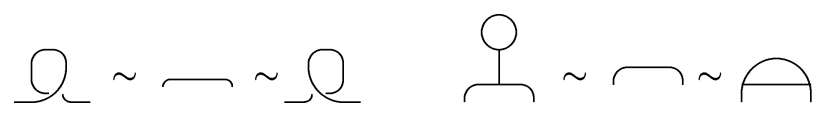

Р ис. 6. Диаграммы зацеплений и соответствующие полихордовые диаграммы

Таким образом, мы получаем либо $n$, либо $n+1$ окружностей.

Если изначально была всего одна окружность, мы видим, что в первом случае первое движение Райдемайстера добавляет отдельно стоящую хорду (или пару скобок [ ] = ( ) $=1$ ); диаграмма при этом остается (моно) хордовой. Это движение мы назовем (1). Второй случай, который из одной окружности делает две, будет рассмотрен позже.

\subsection{2. Второе движение Райдемайстера.}

Лемма 2.4.2.1 (см. рис. 7). При применении второго движения Райдемайстера количество послекритических окружностей либо увеличивается на 1, либо уменьиается на 1 .
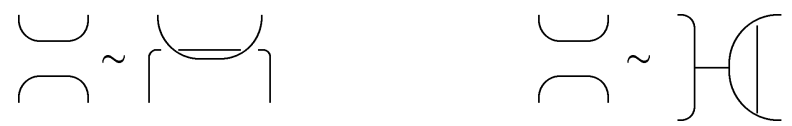

Р ис. 7. Диаграммы зацеплений и соответствующие полихордовые диаграммы

ДокАЗАТЕЛЬСТво. Четыре конца этих параллельных линий могут быть соединены (глобально на полихордовой диаграмме), образуя одну или две окружности.

После второго движения Райдемайстера эти линии перестраиваются так, что количество окружностей, образованных этими линиями, меняется с единицы на двойку и наоборот. Легко видеть, что количество оставшихся окружностей при этом не меняется, что и завершает доказательство леммы.

Таким образом, второе движение Райдемайстера никогда не может оставлять классическую диаграмму классической.

2.4.3. Третье движение Райдемайстера. В некоторых случаях количество окружностей не меняется при третьем движении Райдемайстера; в этом случае соответствующее соотношение на хордовых диаграммах (скобочных структурах) выглядит следующим образом.

Лемма 2.4.3.1. При применении третьего движения Райдемайстера количество окружностей либо остается тем же, либо увеличивается на 2, либо уменьшается на 2 . 
1)

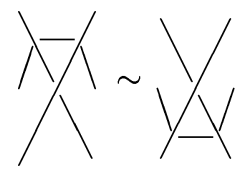

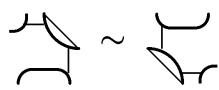

2)

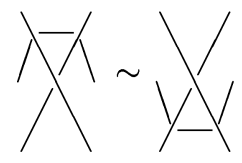

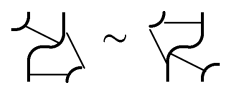

Рис. 8. Слева - плоские диаграммы, справа - полихордовые диаграммы

ДоКАЗАТЕЛЬСТВО заключается в простом рассмотренни этих движений. В случае каждого из них существует шесть концов, которые должны быть соединены друг с другом на плоскости без пересечений, образуя одну, две или три окружности (см. рис. 8).

Легко видеть, что в случае диаграммы 1 (рис. 8), количество окружностей изменяется на 2 , а в случае диаграммы 2 (рис. 8 ) не изменяется, что и завершает доказательство леммы.

2.4.4. Построение системы глобальных соотношений (1)-(6). Итак, пусть дано зацепление $K$ и две его плоские диаграммы $K_{1}$ и $K_{2}$, причем хордовые диаграммы, соответствующие $K_{1}$ и $K_{2}$, являются классическими.

Наша цель - построить цепь преобразований, переводящих $K_{1}$ в $K_{2}$. При этом все промежуточные хордовые диаграммы должны быть (моно)хордовыми.

Мы делаем это следующим образом. Так как $K_{1} \equiv K_{2}$, существует последовательность $K_{1}=A_{1}=A_{2}=\cdots=A_{n}=K_{2}$ плоских диаграмм зацепления $K$. При этом $A_{i+1}$ должно получаться их $A_{i}$ одним движением Райдемайстера. При этом хордовые диаграммы, соответствующие $A_{i}, i=1, \ldots, n$, могут быть, вообще говоря, полихордовыми.

Наша задача - построить с помошью $A_{i}, i=1, \ldots, n$, систему $B_{j}, j=1, \ldots, m$, реализаций зацепления $K$ такую, что

i) все $B_{j}, j=1, \ldots, m$, таковы, что соответствующие им хордовые диаграммы являются классическими;

ii) $B_{j}$ и $B_{j+1}$ отличаются друг от друга “не сильно", т.е. переводятся друг в друга неким глобальным соотношением;

iii) $B_{1}=A_{1}, B_{m}=A_{n}$.

Итак, рассмотрим систему $A_{i}, i=1, \ldots, n$, плоских диаграмм. Пусть $k_{i}-$ количество окружностей на хордовой диаграмме, соответствующей $A_{i}$.

С помошью лемм 2.4.2.1 и 2.4.3.1, описьваюших влияние движений Райдемайстера на количество окружностей соответствующей хордовой диаграммы, мы строим систему движений, оставляющих диаграмму классической.

Идея построения $B_{j}$ состоит в следуюшем: для каждого $A_{i}$ мы строим соответствующее $B_{j}$ (вообще говоря, $j \geqslant i$, это будет показано в дальнейшем), отличающееся от $A_{i}$ $\left(k_{i}-1\right)$-кратным применением второго движения Райдемайстера, которое "соединяет" $k_{i}$ окружностей в одну. Положим $B_{1}=A_{1}$.

ОпРЕДЕЛЕниЕ 2.4.4.1. Рассмотрим некое движение Райдемайстера $R_{1}$, примененное к некоторой классической хордовой диаграмме. Назовем композицию $R_{2}$ одного или нескольких вторых движений Райдемайстера движениями, компенсирующими $A$, если композиция $R_{2} \circ R_{1}$ оставляет диаграмму классической.

Мы строим систему $B$ таким образом, чтобы $B_{j}$ изменялись так же, как $A_{i}$, но с некоторыми добавлениями или убираниями “компенсирующих" вторых движений Райдемайстера. 
В случае, когда на месте движения, “происходящего из $A$ ", стоит препятствие в виде компенсирующего второго движения Райдемайстера, необходимо произвести перебрасывание компенсирующего второго движсения Райдемайстера, т.е. композицию двух вторых движений Райдемайстера, одно из которых увеличивает количество окружностей полихордовой диаграммы на единицу, а второе уменьшает их количество на единицу. В терминах хордовых диаграмм это движение выглядит следуюшим образом (рис. 9):

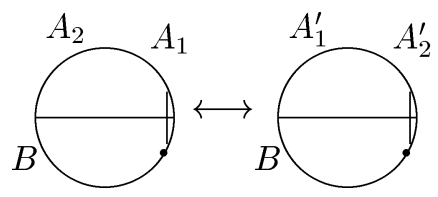

Рис. 9

Мы будем обозначать его через (2) (см. далее).

Так как среди движений, переводящих $B_{i}$ в $B_{i+1}$, встречаются движения типа 2 , то количество движений типа $B$, вообще говоря, больше количества движений типа $A$, т.е. $m \geqslant n$.

Приведем список движений типа $B$ в терминах хордовых диаграмм и правильных двухскобочных структур (как и должно быть, они совпадают с движениями (1)-(7)).

(1) Первое движение Райдемайстера. Добавление отдельно стоящей хорды:

$$
()=[]=1 \text {. }
$$

(2) Перебрасьвание второго движения Райдемайстера (рис. 10):

$$
\text { ( [ ) } \left.A_{1} A_{2}\right]=\left(\left[\text { ) } A_{2} A_{1}\right]\right. \text {, }
$$

где одно из слов $A_{i}, i=1,2$, имеет правильную скобочную структуру относительно $($,$) .$ Значение штриха см. во введении.

(3) Первое движение Райдемайстера, компенсированное вторым:

$$
\text { ( [ ) ( ] } A)=A \text {, }
$$

где слово $A$ имеет правильную относительно $($, ) структуру.

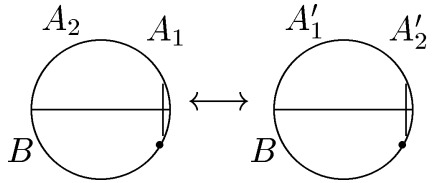

Рис. 10

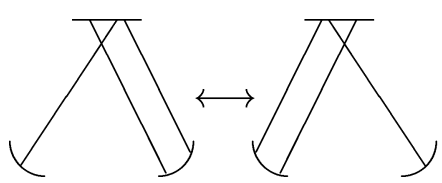

Рис. 11 


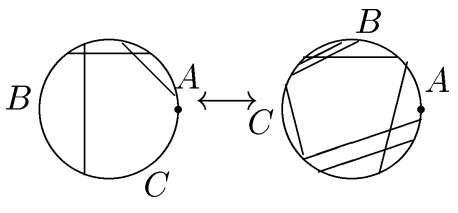

Рис. 12

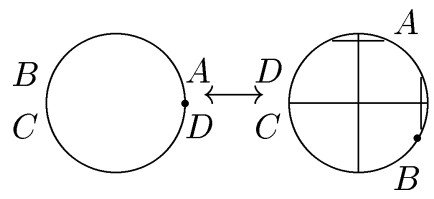

Рис. 13

(4) Третье движение Райдемайстера (случай 2; см. рис. 11):

$$
([(A)) B]=[([A) B]],
$$

где слова $A$ и $B$ имеют правильные (, )-структуры.

(5) Третье движение Райдемайстера (случай 1), компенсированное двумя вторыми движениями Райдемайстера (рис. 12):

$$
A[((] B) C)=A((B[[)]](C)[[\text { ] ] ], }
$$

где слова $A, B, C$ имеют правильные [, ]-структуры.

(6) Добавление (убирание) двух вторых движений Райдемайстера (компенсация одного движения Райдемайстера другим; см. рис. 13). Формула:

$$
A B C D=\left(\text { [ ) } A\left[(] D^{\prime}\right] C^{\prime}\right) B^{\prime},
$$

где слово $A D$ имеет правильную [, ]-структуру, а слово $D C$ - правильную (, )-структуpy. Значение $B^{\prime}, C^{\prime}, D^{\prime}$ см. во введении.

Таким образом, для доказательства главной теоремы осталось проверить условия i)-іii) построения системы $B_{j}$.

Условие i) следует из построения.

Выполнение условия iі) проверяется, поскольку всем глобальным соотношениям $A_{i}$, т.е. всем типам движений Райдемайстера, сопоставлены движения $B_{j}$, и, кроме того, добавлено необходимое "перебрасывание".

Проверим условие iii). Равенство $B_{1}=A_{1}$ верно по построению. Выполнение условия $B_{m}=A_{n}$ следует из того, что хордовая диаграмма, соответствующая $A_{n}$, является классической, т.е. $k_{n}=1$. Таким образом, количество "компенсирующих" вторых движений Райдемайстера, отличающих $B_{m}$ от $A_{n}$, равно нулю.

Пусть дана правильная двухскобочная структура $D$. Рассмотрим следующее множество скобок из $D$ : первую скобку $A_{1}$ (открывающуюся), скобку $A_{2}$, стоящую после скобки, закрьвающей $A_{1}$ (будем считать, что после последней скобки идет первая), скобку $A_{3}$, стоящую перед скобкой, соответствующей $A_{2}$, и т.д. Скобка $A_{n}$ стоит перед скобкой, соответствующей $A_{n-1}$, при $n$ нечетном и после нее при $n$ четном. Назовем $a_{i}$ пару соседних скобокб т.е. скобки, соответствуюшие $A_{i}$ и $A_{i+1}$.

ОПРЕДЕЛЕНИЕ 2.4.4.2. Назовем правильную двухскобочную структуру $D$ хорошей, если $a_{i}$ не повторяются до тех пор, пока все пары соседних скобок не пройдены.

ПримеР. Структура $\left[a_{8}\left[a_{5}\left(a_{2}\left(a_{7}\right] a_{4}\right] a_{1}\right) a_{6}\right) a_{3}$ хорошая, а структура $\left(a_{4}\left[\left[a_{2}[) a_{1}\right]\right] a_{3}\right]$ нет.

Легко видеть, что хорошие правильные двухскобочные структуры кодируют узлы, т.е. зацепления с одной компонентой ( $a_{i}$ показывает обход узла). При этом соотношения (1)-(7), описанные во введении, оставляют узел узлом. Таким образом, получаем следствие. 
СлЕДСТвИЕ 2.4.4.1. Подполугругруппа $G^{\prime} \subset G$ классов әквивалентности хороших правильных двухскобочных структур по глобальным соотношениям (1)-(7) изоморфна подполугруппе $K^{\prime} \subset K$ всех узлов (однокомпонентных зачеплений) $c$ классом тривиального узла в роли единищы и умножсением, индуцированным из $K$.

\section{§3. Виртуальный случай}

3.1. Основные определения. В 1996 году Л. Кауффманом было дано определение виртуального узла (имеется ввиду узел или зацепление) - естественное обобщение понятия узла, основанное на понятии диаграммы узла.

ОПРЕДЕЛЕНИЕ 3.1.1. Диаграммой виртуального зацепления называется плоский граф, все вершины которого имеют кратность 4; при этом в каждой вершине может быть проход, переход и так называемоевиртуальное пересечение, которое изображается символически в виде, указанном на рис. 14.

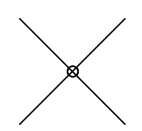

Рис. 14. Виртуальное пересечение

Две диаграммы назьваются әквивалентными, если существует последовательность так назьваемых виртуальных движений Райдемайстера, переводящих первую диаграмму во вторую.

ОПРЕДЕЛЕНИЕ 3.1.2. Приводим список виртуальных движений Райдемайстера.

1.Классические движения Райдемайстера, относящиеся к классическим вершинам.

2. Виртуальные версии классических движений Райдемайстера с заменой классических вершин на виртуальные (см. рис. 15).

3. Версия третьего “треугольного” движения Райдемайстера с заменой двух реальных вершин на дуге, “проходящей” через третью вершину, на виртуальные (см. рис. 16).

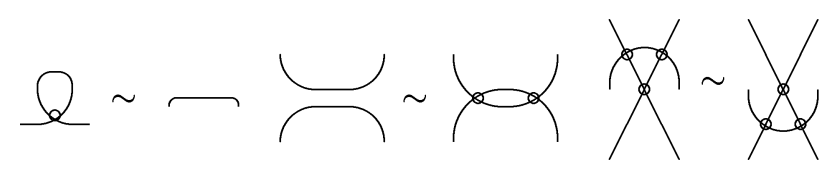

Рис. 15

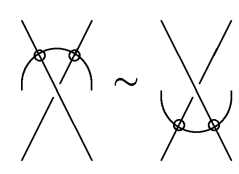

Рис. 16

ОПРЕДЕЛЕНИЕ 3.1.3. Виртуальным зацеплением называется класс эквивалентности виртуальных диаграмм по виртуальным движениям Райдемайстера.

ОПРЕДЕЛЕНИЕ 3.1.4. Пусть дан ориентируемьй атом $V$ с фиксированной согласованной ориентацией послекритических окружностей. Назовем плоской реализацией aтома $V$ гладкое погружение остова атома в плоскость такое, что

i) если точка на плоскости имеет более одного прообраза, то она является точкой трансверсального пересечения ровно двух образов линий;

ii) сохранена структура $A$ атома $V$ в каждой вершине (определение см. в 2.1), кроме того, образ каждой послекритической окружности в каждой вершине локально проходит по часовой стрелке (см. рис. 17). 

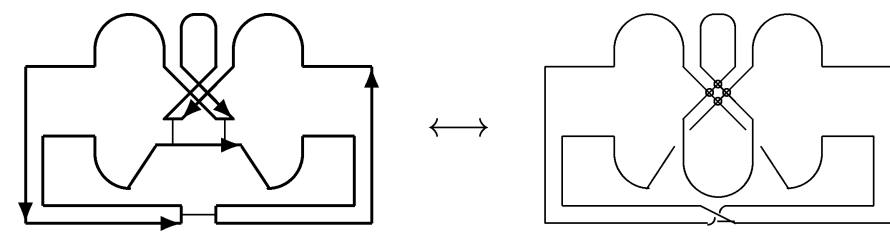

Рис. 17. Слева - иммерсия (поли)хордовой диаграммы ориентируемого атома, справа - диаграмма виртуального узла

\section{2. Ориентируемые атомы и “ориентируемые" виртуальные зацепле-} ния. Пусть дан ориентируемый атом $V$ и его плоская реализация. Поставим ему в соответствие диаграмму виртуального зацепления: в каждом образе вершины атома ставим проход или переход так же, как в случае высотных атомов и классичеких зацеплений; в точках пересечения образов двух ребер ставим виртуальное пересечение.

ТЕорема 3.2.1 (обобщенная теорема об атомах и узлах). Пусть дан ориентируемый атом $V$. Тогда любой его плоской реализации соответствует диаграмма одного и того же виртуального зачепления.

ДокАЗАТЕЛЬСТво основывается на том факте, что переход от одной реализации к другой можно осуществить с помощью движений, соответствующих виртуальньп движениям Райдемайстера.

ОПРЕДЕЛЕНИЕ 3.2.1. Пусть дан ориентированньй атом $V$. Назовем виртуальное зацепление, построенное с помощњю плоской реализации $V$, зацеплением, соответствующим атому $V$.

ОПрЕдЕлЕниЕ 3.2.2. Назовем все виртуальные зацепления, соответствующие хотя бы одному ориентируемому атому, ориентируемыми виртуальными зацеплениями.

Пусть дано виртуальное зацепление $K$. Рассмотрим его различные диаграммы и построим по ним атомы (в каждой реальной вершине ставим вершину атома с соответствующей $B$-структурой, в каждой виртуальной вершине не ставим ничего - это просто пересечение двух ребер атома). Заметим, что полученны атом может и не быть ориентируемьми.

ТЕОРемА 3.2.2. Четность әйлеровой характеристики атома на меняется при виртуальных движениях Райдемайстера, тем самым она является инвариантом виртуального зачепления.

ДокАЗАТЕЛЬСТВО состоит в аккуратном рассмотрении всех виртуальных движений Райдемайстера.

ПРИМЕР НЕОРИЕНТИРУЕМОГО УЗЛА. Рассмотрим виртуальньй узел, показанный на рис. 18.

Атом, соответствуюший данной диаграмме, лежит на проективной плоскости, следовательно имеет эйлерову характеристику 1 . Отсюда следует, что данное виртуальное зацепление не является ориентируемым.

\section{3. Ориентируемые виртуальные зацепления и хордовые диаграммы.}

Вспомним, что у каждого ориентированного атома есть полихордовая диаграмма, т.е. $f$-граф с согласованно ориентированными окружностями без меток. 


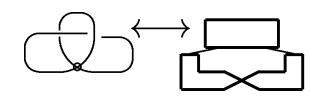

Рис. 18. $\mathrm{B}=2, \mathrm{P}=4, \Gamma=3 ; \mathrm{B}-\mathrm{P}+\Gamma=1$

Теорема 3.3.1. Для каждого ориентируемого виртуального зацепления $K$ существует соответствующий ориентируемый атом $V$ такой, что полихордовая диаграмма, соответствующая $V$, является диаграммой с одной окружностью.

ДоКАЗАТЕЛЬСТВО полностью аналогично доказательству теоремы 2.2.2 и заключается в многократном исправлении данной диаграммы с несколькими окружностями с помощью вторых обычных движений Райдемайстера, при каждом из которых количество окружностей уменьшается на единицу, а в конще становится равным единище.

Тем самым, множество всех хордовьх диаграмм кодирует все ориентируемые виртуальные зацепления, при этом можно построить систему соотношений на хордовых диаграммах, кодируюших одно и то же виртуальное зацепление, аналогично тому, как это сделано для классических зацеплений. Хордовые диаграммы, эквивалентные $d$-диаграммам, кодируют классы классических зацеплений.

Однако, пока не найдена кодировка полугрупшы всех хордовых диаграмм по некоторьм соотношениям, кодирующая все ориентируемые виртуальные зацепления с помощью слов в некотором конечном алфавите.

Автор выражает благодарность В.А. Васильеву за поддержку и внимание к работе, а также А.Б. Сосинскому и С.К. Ландо за внимание к работе.

\section{СПИСОК ЦИТИРОВАННОЙ ЛИТЕРАТУРЫ}

[1] Мантуров В. О. Атомы, высотные атомы, хордовые диаграммы и узлы. Перечисление атомов малой сложности с использованием языка Mathematica 3.0 // Топологические методы в теории гамильтоновых систем / ред. А. Т. Фоменко, А. В. Болсинов, А. А. Шафаревич, 1998. C. $203-212$.

[2] Мантуров В.О. Бифуркации, атомы и узлы // Вестн. МГУ. Сер. 1. Матем., мех. 2000. №1. C. $1-7$.

[3] Прасолов В. В., Сосинский А. Б. Узлы, зацепления, косы и трехмерные многообразия: Изд. МЦНМО, 1997.

[4] Fomenko A.T. The theory of multidimensional integrable hamiltonian systems (with arbitrary many degrees of freedom). Molecular table of all integrable systems with two degrees of freedom // Adv. Soviet Math. 1991. V. 6. P. 1-35.

[5] Ошемков А. А. Функции Морса на двумерных поверхностях. Кодирование особенностей // Тр. МИРАН. 1994. Т. 205. С. 131-141.

[6] Bar-Natan D. On the Vassiliev knot invariants // Topology. 1995. V. 34. P. 423-475.

Московский государственный университет им. М.В. Ломоносова

Поступило

E-mail : manturov@matov.msk.ru

31.08.1999

Исправленный вариант

22.10 .1999 